\title{
Perioperative Management of Obstructive Sleep Apnea With Nasal Continuous Positive Airway Pressure
}

\author{
Hirofumi Arisaka, MD, DDS, PhD, * Shigeki Sakuraba, MD, PhD, $†$ Reiko Kobayashi, DDS, * \\ Homare Kitahama, DDS, ${ }^{*}$ Naofumi Nishida, DDS, ${ }^{*}$ Munetaka Furuya, DDS, PhD, ${ }^{*}$ and \\ Kazu-ichi Yoshida, DDS, PhD*
}

*Division of Anesthesiology, Department of Clinical Care Medicine, Kanagawa Dental College, Kanagawa, Japan, and †Department of Anesthesiology, School of Medicine, Keio University, Tokyo, Japan

The high risks associated with general anesthesia in obstructive sleep apnea syndrome (OSAS) patients have been reported. Many authors have suggested that the intraoperative administration of opioids and sedatives should be limited or avoided because these drugs selectively impair muscle activity in the upper airway. We report the case of an OSAS patient who was managed with nasal continuous positive airway pressure (NCPAP) and treated safely in spite of the use of conventional anesthetic and analgesic agents typically used for patients without OSAS. She had little pain during the perioperative period. It is suggested that NCPAP is an effective treatment for not only preventing airway obstructive apnea but for allowing the administration of anesthetic and analgesic drugs without major complications.

Key Words: Obstructive sleep apnea syndrome; NCPAP; Perioperative management.

$\mathbf{M}$ any reports concerning anesthetic management in patients with obstructive sleep apnea syndrome (OSAS) have been addressed. ${ }^{1-7}$ These underline the higher risks associated with general anesthesia in OSAS patients. Those risks of perioperative complications might cause physicians to limit their administration of pain and anxiety relief drugs in the perioperative period. We report the case of an OSAS patient who was managed with nasal continuous positive airway pressure (NCPAP) and treated safely with anesthetic and analgesic drugs.

This case may suggest that NCPAP not only prevents airway obstruction, but also allows the administration of anesthetic and analgesic drugs without major complication.

Received May 31, 2007; accepted for publication July 17, 2008.

Address correspondence to Dr Hirofumi Arisaka, 82 Inaoka-cho, Yokosuka, Kanagawa 238-8580, Japan; a-hiroumi@mpd.biglobe.ne.jp.

Anesth Prog 55:121-123 2008

(C) 2008 by the American Dental Society of Anesthesiology

\section{CASE REPORT}

The patient was a 66-year-old woman, weight $60 \mathrm{~kg}$, height $158 \mathrm{~cm}$, body mass index (BMI) $24.0 \mathrm{~kg} / \mathrm{m}^{2}$. She experienced episodes of severe apnea and oxygen desaturation in the intensive care unit (ICU) following uterine myomectomy at the age of 48 years and right mastectomy at the age of 53 years. These episodes, a long history of loud snoring, and daytime somnolence indicated the possibility of OSAS, a condition that may have not been fully diagnosed at the times of previous operations. Two weeks before the left mastectomy was scheduled, OSAS was diagnosed following a full-night polysomnography (PSG) conducted at Kanagawa Dental College Hospital.

The results of the PSG indicated that the patient had severe OSAS, with an apnea hypopnea index (AHI) of 49 and a minimum oxygen saturation of $80 \%$ (Table). A regimen of NCPAP (REMstar Auto, Respironics Inc) was started 1 week prior to the operation, and the pressure was adjusted to enable her to 
Polysomnography: Clinical Reports From Our Case*

\begin{tabular}{lc}
\hline TST & $463.0 \mathrm{~min}$ \\
REM & $17.04 \%(20-25 \%)$ \\
Non-REM Stage 1 & $29.3 \%(2-5 \%)$ \\
Non-REM Stage 2 & $53.3 \%(45-55 \%)$ \\
Non-REM Stage 3 + Stage 4 & $0 \%(13-23 \%)$ \\
Arousal index & $39.5 / \mathrm{h}$ \\
AHI & $48.7 / \mathrm{h}$ \\
AI & $18.7 / \mathrm{h}$ \\
Obstructive apnea & $18.7 / \mathrm{h}$ \\
$\quad$ Central apnea & $0 / \mathrm{h}$ \\
Mixed apnea & $0 / \mathrm{h}$ \\
Desaturation index $\dagger$ & $42.8 / \mathrm{h}$ \\
Minimum saturation $(\%)$ & $80 \%$ \\
Saturation $<90 \%$ & $44.5 \mathrm{~min}$ \\
Saturation $<85 \%$ & $5.0 \mathrm{~min}$
\end{tabular}

* TST indicates total sleep time; REM, rapid eye movement; Arousal index, sleep fragmentation index; AHI, apnea hypopnea index (showing that an AHI of 5 to 15 represents mild sleep apnea, 15 to 30 represents moderate sleep apnea, and greater than 30 represents severe sleep apnea); AI, apnea index.

t The oxygen desaturation index is calculated as the number of $4 \%$ dips in $\mathrm{SaO}_{2}$ per hour of sleep.

tolerate NCPAP while asleep. NCPAP pressure was $8 \mathrm{~cm} \mathrm{H}_{2} \mathrm{O}$.

\section{Intraoperative Management}

The patient received no premedication. Standard monitoring was performed, including noninvasive blood pressure, heart rate from electrocardiography (lead II), and oxygen saturation by pulse oximetry. Anesthesia was induced by propofol, $150 \mathrm{mg}$, and fentanyl, $100 \mu \mathrm{g}$. Intubation of the trachea was performed without difficulty following intravenous administration of vecuronium, $6.0 \mathrm{mg}$. Anesthesia was maintained with oxygen, nitrous oxide, sevoflurane, and fentanyl, $200 \mu \mathrm{g}$. The operation lasted 4 hours. The intraoperative course was uneventful and hemodynamics remained stable throughout the surgery. The patient was extubated in the operating room. During her emergence, she complained of little pain.

\section{Postoperative Management}

The patient was taken to the ICU and immediately started on NCPAP. Room air was initially supplemented by $4 \mathrm{~L} / \mathrm{min}$ of oxygen via the intake port of the mask. She was administered NCPAP for all sleep periods thereafter for the duration of her stay in the ICU. She experienced no episodes of apnea or desaturation in the ICU and complained of little pain with analgesic agents of pentazocine, $15 \mathrm{mg}$, and hydroxyzine, $25 \mathrm{mg}$. Her recovery was uneventful and she was discharged from the hospital 8 days after her operation.

\section{DISCUSSION}

Although many reports have addressed anesthetic management in patients with OSAS, there are currently few studies, no formalized clinical practice guidelines, and insufficient evidence to guide the clinical decision-making of anesthesiologists in the perioperative care of patients with OSAS. ${ }^{8}$

Patients with obstructive sleep apnea present challenges to the anesthesiologist throughout the perioperative period. ${ }^{5,6}$ During this period, one must remain aware of the proclivity of obstructive sleep apnea patients to develop acute upper airway obstruction after minimal sedation. Hypoglossal nerve activity is very sensitive to minimal levels of anesthesia, and the genioglossus muscle tone, which maintains upper airway patency, may be lost. ${ }^{2,9}$ The use of these drugs may increase the number and duration of apneic eposides, which are associated with decreases in oxygen saturation. ${ }^{3}$ It is, therefore, recommended that no sedatives be given preoperatively. ${ }^{2,9}$ Similarly, extreme care must be taken in the management of patients with OSAS in the intraoperative period. Many authors have suggested that the intraoperative administration of opioids and sedatives should be limited or avoided. ${ }^{3-6}$ Esclamado et $\mathrm{al}^{7}$ in their retrospective study identifying specific risk factors for sleep apnea patients, noted that the amount of opioid administered intraoperatively correlated with extubation problems (ie, airway-obstructive apnea after extubation), as was highlighted by a comparison of fentanyl doses $(2.9 \mu \mathrm{g} / \mathrm{kg}$ in the complications group versus $1.7 \mu \mathrm{g} / \mathrm{kg}$ in the group that did not experience complications). In our case, an intraoperative dose of fentanyl, $5 \mu \mathrm{g} / \mathrm{kg}$, was administered, but the patient did not experience airway obstruction or other postoperative complications including pain, which we attribute to respiratory management with NCPAP.

The risk of airway obstruction is highest in the postoperative period, making the recovery period extremely important for sleep apnea patients. ${ }^{9}$ It has been suggested that patients with sleep apnea be kept in the recovery room for an extended period to monitor respiration and cardiac function. ${ }^{1}$ Careful postoperative monitoring for apnea, oxygen desaturation, and dysrhythmias in ICU is a necessity. Sleep apnea may result not only in hypoxia, but also systemic and pulmonary hypertension, cardiac arrhythmias, myocardial infarction, ${ }^{10}$ and interruptions of sleep with decreases of stage 3 and 4 non-REM sleep. Unexpected mortality still occurs in this patient group following major surgical procedures. The majority of incidents of 
postoperative cardiac ischemia, myocardial infarction, and sudden cardiac death occur at night. It has been suggested that OSAS may be a major cause of these unexpected cases of postoperative cardiac ischemia or death. ${ }^{11,12}$

NCPAP is an effective treatment for obstructive sleep apnea. Despite the patient's diagnosis of severe obstructive sleep apnea, she did not experience airway obstruction or desaturation in the postoperative period when nasal CPAP was maintained up to the time of discharge. We suggest that a regimen of NCPAP initiated prior to surgery and resumed immediately after extubation may allow patients to be treated safely with conventional anesthetic and analgesic agents, and that OSAS patients who receive NCPAP may follow perioperative courses comparable to those of patients without OSAS. ${ }^{13}$

In our case, although the patient had experienced episodes of heavy apnea and oxygen desaturation in the ICU following 2 previous operations, NCPAP prevented apnea and desaturation following the operation described in this case, and little pain was observed owing to the administration of fentanyl and analgesic agents.

A substantial proportion of OSAS patients will not have been previously diagnosed, as OSAS is a notoriously under-diagnosed condition. ${ }^{14}$ Many patients might remain undiagnosed OSAS until postoperative events occur, like frequent or severe airway obstruction or hypoxemia events. The present paper suggests that the initiation of nasal CPAP should be considered. However, where CPAP is refused and upper airway obstruction continues to be problematic, use of lateral positioning, a nasopharyngeal airway, and oxygen therapy should also be considered. However, every effort should be made to identify patients with OSAS preoperatively and to institute NCPAP therapy prior to surgery. It is suggested that NCPAP is an effective treatment for obstructive sleep apnea that not only prevents airway obstruction and the other consequences of OSAS, but also allows the administration of anesthetic and analgesic drugs without major complication. In conclusion, the patients being treated for OSAS should have their CPAP restarted immediately in the postoperative period. This perioperative management of the OSAS patient can be applied to anesthetic management of the OSAS patient during oral and dental treatment.

In comparison with full face CPAP, which covers both the nose and mouth, nasal CPAP covers only the nose. Nasal CPAP rather than full-face mask CPAP may be a good option for the patient undergoing oral surgery where access to bleeding areas, gauze packing, suctioning, and so on is necessary postoperatively. Tissue damage, edema, bleeding, swelling, and hematomas caused by oral and dental surgery may al- so induce postoperative airway obstruction. In such a case, the application of CPAP alone is inappropriate, as prolonged tracheal intubation or tracheotomy may be required. Therefore, we should discuss the various anesthetic aspects of obstructive sleep apnea, including preoperative, perioperative, and postoperative points of special interest. In oral and dental surgery, we should judge OSAS patients on a case-by-case basis and consider the various management options.

\section{REFERENCES}

1. Chung F, Crago RR. Sleep apnoea syndrome and anaesthesia. Can Anaesth Soc J. 1982;29:439-445.

2. Rafferty TD, Ruskis A, Sasaki C, Gee JB. Perioperative considerations in the management of tracheotomy for the obstructive sleep apnoea patient. Three illustrative case reports. BrJ Anaesth. 1980;52:619-622.

3. Hanning CD. Obstructive sleep apnoea. BrJ Anaesth. 1989;63:477-488.

4. Connoly LA. Anaesthetic management of obstructive sleep apnea patients. $J$ Clin Anesth. 1991;3:461-469.

5. Glenn MG, McCulloch TM, Cummings CW. Perioperative complications and risk factors in the surgical treatment of obstructive sleep apnea syndrome. Laryngoscope. 1989;99:1125-1129.

6. VanDercar DH, Martinez AP, De Lisser EA. Sleep apnea syndromes: a potential contraindication for patient-controlled analgesia. Anesthesiology. 1991;74:623-624.

7. Esclamado RM, Glenn MG, McCulloch TM, Cummings CW. Perioperative complications and risk factors in the surgical treatment of obstructive sleep apnea syndrome. Laryngoscope. 1989;99:1125-1129.

8. Turner K, VanDenkerkhof E, Lam M, Mackillop W. Perioperative care of patients with obstructive sleep apnea-a survey of Canadian anesthesiologists. Can J Anaesth. 2006;53:299-304.

9. Keamy MF III, Cadieux RJ, Kofke WA, Kales A. The occurrence of obstructive sleep apnea in a recovery room patient. Anesthesiology. 1987;66:232-234.

10. Shepard JW Jr. Hypertension, cardiac arrhythmias, myocardial infarction, and stroke in relation to obstructive sleep apnea. Clin Chest Med. 1992;13:437-458.

11. Reeder MK, Muir AD, Foex P, Goldman MD, Loh L, Smart D. Postoperative myocardial ischaemia: temporal association with nocturnal hypoxaemia. $\mathrm{Br} J$ Anaesth. 1991;67:626-631.

12. Rosenberg J, Pedersen MH, Ramsing T, Kehlet H. Circadian variation in unexpected postoperative death. BrJ Surg. 1992;79:1300-1302

13. Rennotte MT, Baele P, Aubert G, Rodenstein DO. Nasal continuous positive airway pressure in the perioperative management of patients with obstructive sleep apnea submitted to surgery. Chest. 1995;107:367-374.

14. Hillman DR, Loadsman JA, Platt PR, Eastwood PR. Obstructive sleep apnoea and anaesthesia. Sleep Med Rev. 2004;8:459-471. 